\title{
Acute postoperative pain and opioid consumption after laparoscopic cholecystectomy is associated with body mass index: a retrospective observational single-center study
}

\author{
@Ößkkeş Hakan Miniksar¹, @Mehmet Kağan Katar² \\ ${ }^{1}$ Yozgat Bozok University, Faculty of Medicine, Department of Anesthesiology and Reanimation, Yozgat, Turkey \\ ${ }^{2}$ Atlas University, Faculty of Medicine, Department of General Surgery, İstanbul, Turkey
}

Cite this article as: Miniksar ÖH, Katar MK. Acute postoperative pain and opioid consumption after laparoscopic cholecystectomy is associated with body mass index: a retrospective observational single-center study. J Health Sci Med 2022; 5(1): 1-6.

\begin{abstract}
Aim: The aim of this study was to determine the relationship between postoperative pain scores and opioid analgesic consumption according to BMI levels in patients undergoing laparoscopic cholecystectomy.

Material and Method: In this retrospective observational study, we scanned the medical data of 154 patients aged from 18-55 years who underwent laparoscopic cholecystectomy. Patients were divided into two groups based on the BMI cut-off value (Group Non-obese, BMI $<30 \mathrm{~kg} / \mathrm{m}^{2}, \mathrm{n}=59$; Group Obese, BMI $>30 \mathrm{~kg} / \mathrm{m}^{2}, \mathrm{n}=35$ ). Postoperative visual analog scale (VAS) pain scores, total tramadol consumption, and intraoperative fentanyl bolus requirements were compared between the groups at fivetime points (T0: in the recovery room, T1: 1st hour in the ward, T2: 6th hour, T3: 12th hour, and T4: 24th hour).

Results: Postoperative VAS pain scores were significantly higher in the Group Obese at T1-2 time points $(\mathrm{p}=0.009)$. The number of patients with a VAS score of $>3$ at the T- 0 time point was significantly higher in the Group Obese ( $\mathrm{p}=0.014)$. Total tramadol consumption was significantly higher in the Group Obese $(40.0 \pm 46.6 \mathrm{mg})$ than in the Group Non-obese $(16.10 \pm 34.0$ $\mathrm{mg})(\mathrm{p}=0.003)$. There was a weak positive correlation between BMI and postoperative pain scores (T0, T-1, T-2) of the patients, and a moderate positive correlation $(\mathrm{r}=0.307)$ between total tramadol consumption.

Conclusions: According to the results of this study, BMI is associated with acute postoperative pain in patients undergoing cholecystectomy, and obese patients require more opioid analgesia postoperatively. However, postoperative analgesia requirements should be determined according to BMI levels in patients undergoing cholecystectomy.
\end{abstract}

Keywords: Obesity, postoperative pain, analgesia, opioid consumption, laparoscopy

\section{INTRODUCTION}

Cholecystectomy is one of the most common intra-abdominal surgeries, usually performed laparoscopically. Although acute postoperative pain is less common in laparoscopic surgery compared to open surgery, many patients (30-70\%) complain of moderate to severe pain (1).

Acute postoperative pain can be defined as pain that occurs due to a surgical procedure and decreases tissue healing over time. Many studies have reported an increase in length of stay, sleep disturbance, decrease in patient satisfaction, prolonged immobilization time and increase in opioid analgesic consumption due to poor control of postoperative pain (2). It may also cause the development of chronic pain (1-3). Therefore, it is important to treat it effectively (3).
Many factors such as the presence of preoperative pain, preoperative anxiety, applied analgesic treatment method, depression, surgical procedure, inflammation, neuronal damage, gender, smoking, obesity and age play a role in the etiology of postoperative pain (4). Therefore, it is difficult to fully assess postoperative pain and apply a standard treatment. Although the cause of postoperative pain is inflammation and neuronal damage, its pathophysiology is typical and outcomes vary according to the patient. For this reason, it is necessary to obtain new information on pain mechanisms to identify patients at high risk of postoperative pain in the preoperative period and to develop individual treatment strategies (1-4). 
The relationship between overweight and pain has been the subject of many studies. A high body mass index (BMI) has been associated with many types of pain, such as general body pain, back pain, and migraine (5). In a comprehensive study, it was reported that higher BMI was predictive of poor postoperative pain control (6). Conversely, in another study, it was reported that there was no relationship between BMI and pain (7). However, the relationship between body mass index and postoperative pain is still controversial. Therefore, the potential relationship between overweight or obesity and postoperative pain needs to be investigated by further clinical studies. In addition, determining the relationship between preoperative BMI levels and postoperative acute pain scores and opioid consumption in patients who will undergo laparoscopic cholecystectomy (LC) surgery may help clinicians' pain management strategies.

We hypothesized that BMI correlated with postoperative pain scores and opioid analgesic consumption. Therefore, the main aim of this study was to evaluate postoperative pain scores and opioid analgesic consumption, according to BMI levels in patients who underwent LC. The secondary aim was to determine the correlation of BMI levels with postoperative pain scores and opioid analgesic consumption.

\section{MATERIAL AND METHOD}

\section{Study Design}

This retrospective observational single-center study was conducted out at Yozgat Bozok University Medical Faculty Hospital, a tertiary medical center. The study was approved by the Yozgat Bozok University Clinical Researchs Ethics Committee (Date: 27.01.2021, Decision No: KAEK189_2021.01.27_05) and the Helsinki Declaration guidelines were followed throughout the study. The requirement for written informed consent was waived.

\section{Study Participants}

In this study, 154 adult patients of American Society of Anesthesiologist (ASA) physical status 1-3 and over 18 years of age who underwent elective LC between May 2018 and July 2020 were examined. Demographic characteristics of patients, ASA physical status, duration of operation, BMI, and other data were obtained retrospectively from evaluation forms recorded in the anesthesia polyclinic during the preoperative period, anesthesia follow-up forms, patient files, and the hospital information management system. Patients receiving known pain treatment, pregnancy, patients with ASA-IV, undergoing another invasive procedure in addition to LC, patients with an additional neurological and psychiatric history, intraoperative conversion to open cholecystectomy, emergency cases, intraoperative and postoperative non-protocol analgesic applied and records (intraoperative, PACU or ward) missing were excluded from the study. A total of 94 patients remained included in the study (Figure 1).

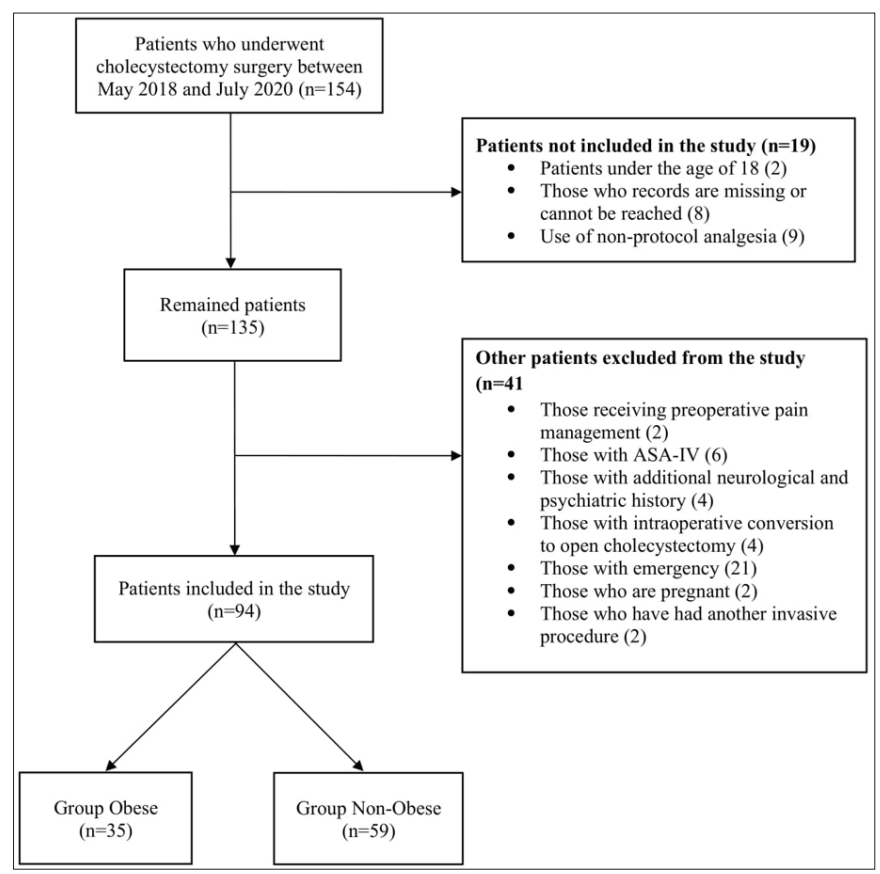

Figure 1. Flow diagram of the study

\section{Clinical Data}

Patients were classified according to their BMI scores as Group Obese $\left(B M I>30 \mathrm{~kg} / \mathrm{m}^{2}\right)$ and Group NonObese $\left(\mathrm{BMI}<30 \mathrm{~kg} / \mathrm{m}^{2}\right)$. During the operation, standard institutional anesthetic induction, monitoring, and maintenance protocol were followed in the study. All patients received standardized anesthesia induction (2-3 $\mathrm{mg} / \mathrm{kg}$ propofol, $0.6-1.2 \mathrm{mg} / \mathrm{kg}$ rocuronium and $1 \mu \mathrm{g} /$ $\mathrm{kg}$ fentanyl). After orotracheal intubation, patients were set to maintain a tidal volume of $8 \mathrm{~mL} / \mathrm{kg}$, I: E ratio 1:2, respiratory rate $12 / \mathrm{min}, \mathrm{ETCO}_{2} 30$ to $40 \mathrm{mmHg}$ in controlled ventilation mode. Maintenance of anesthesia was provided by inhalation of $60 \% \mathrm{O}_{2}$ and $40 \%$ air and sevoflurane (minimum alveolar concentration [MAC] 0.81.3). All surgeries were performed by experienced general surgeons. All patients were given $1 \mathrm{mg} / \mathrm{kg}$ tramadol IV and $1 \mathrm{~g}$ paracetamol IV for postoperative analgesia, according to the pain protocol of our institution, approximately 30 minutes before the end of the operation. At the end of the surgery, residual neuromuscular blockade was antagonized with neostigmine and atropine. When the patient was awake, the endotracheal tube was extubated and the patients were transported to the recovery room. For postoperative pain management, all patients were administered three times a day IV dexketoprofen trometamol $50 \mathrm{mg}$ as a standard. If sufficient analgesia was not provided, in the first step, tramadol $50 \mathrm{mg}$ iv was administered. The total tramadol consumption of patients in the postoperative first 24 hours was recorded. 
The additional dose of fentanyl administered to the patients intraoperatively, the VAS (visual analog scale) (0-10) pain scores in the postoperative recovery unit, the number of patients with VAS $>3 / 10$, and the presence of nausea and vomiting were recorded according to the anesthesia followup form. In the first 24 hours postoperatively, the followup parameters were recorded by examining the nurse observation form. The data were analyzed retrospectively at five-time points in the postoperative period: T0: recovery room, T1: 1st hour in the ward, T2: 6th hour in the ward, T3: 12th hour in the ward, and T4: 24th hour in the ward (Figure 2$)$. VAS resting pain scores $(0=$ no pain and $10=$ worst possible pain), additional opioid analgesic amounts, and presence of nausea-vomiting at all postoperative time points (T0-T4) of the patients were recorded by examining the patient follow-up forms. All these variables were compared between obesity groups.

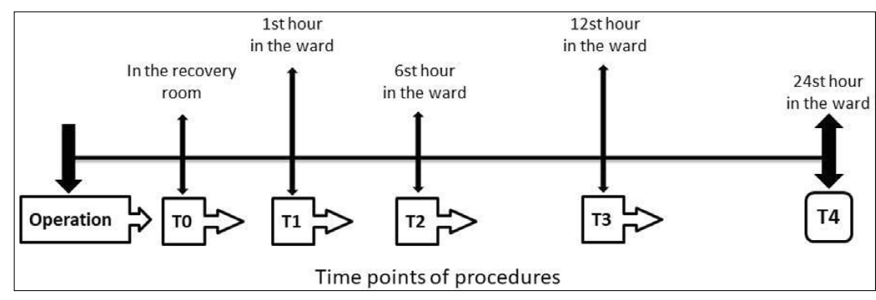

Figure 2. Time points of procedures

\section{RESULTS}

\section{Analysis of Clinical Variables by Obesity Groups}

Postoperative VAS pain scores were higher in the obese group at T1-2 time points and showed a significant difference $(p=0.009)$ (Table 1$)$. There was no statistically significant difference between the groups at other time points $(\mathrm{p}>0.05)$. The number of patients with a VAS score of $>3$ at the T-0 time point was significantly higher in the obese group ( $\mathrm{p}=0.014) .19(54.3 \%)$ patients in the obese group and $17(28.8 \%)$ patients in the non-obese group required additional boluses of fentanyl intraoperatively $(p=0.039)$. Total tramadol consumption was found to be $16.10 \pm 34.0$ significantly higher in the obese group than in the $40.0 \pm 46.6$ non-obese group $(\mathrm{p}=0.003)$ (Table 2). The nausea was observed in $9(25.7 \%)$ patients in the obese group and $6(10.2 \%)$ patients in the non-obese group $(p=0.047)$. Vomiting was observed in 5.3\% (5/94) of the patients. There was no significant difference between the groups in terms of postoperative vomiting.

\section{Correlation Analysis of BMI and Clinical Variables}

There was a weak positive correlation between BMI and postoperative pain scores (T0, T-1, T-2) of the patients, and a moderate positive correlation $(r=0.307)$ with total tramadol consumption (Table 3 ).

\begin{tabular}{|c|c|c|c|c|}
\hline & Group Non-obese $(n=59)$ & Group Obese $(\mathbf{n}=35)$ & Total $(n=94)$ & P value \\
\hline Age (years) & $37.5 \pm 4.0$ & $38.9 \pm 3.8$ & $38.1 \pm 3.9$ & $0.105^{\mathrm{a}}$ \\
\hline Gender, n (\%) & & & & $0.142^{\mathrm{b}}$ \\
\hline Male & $24(40.7)$ & $9(25.7)$ & $33(35.1)$ & \\
\hline Female & $35(59.3)$ & $26(74.3)$ & $61(64.9)$ & \\
\hline ASA physical state, I/II/III (\%) & 25/29/5 (42.4/49.2/8.5) & $10 / 20 / 5(28.6 / 57.1 / 14.3)$ & $35 / 49 / 10(37.2 / 57.1 / 10.6)$ & $0.352^{\mathrm{b}}$ \\
\hline Weight (kg) & $66.8 \pm 7.7$ & $90.4 \pm 13.3$ & $75.6 \pm 15.2$ & $<0.001^{\mathrm{a}}$ \\
\hline Height (m) & $1.8 \pm 0.8$ & $1.6 \pm 0.1$ & $1.7 \pm 0.1$ & $0.001^{\mathrm{a}}$ \\
\hline BMI $\left(\mathrm{kg} / \mathrm{m}^{2}\right)$ & $25.9 \pm 1.7$ & $36.4 \pm 2.6$ & $29.8 \pm 5.5$ & $<0.001^{\mathrm{a}}$ \\
\hline Operation time (minutes) & $72.1 \pm 10.4$ & $71 \pm 12.7$ & $71.7 \pm 11.3$ & $0.631^{\mathrm{a}}$ \\
\hline
\end{tabular}

\begin{tabular}{|c|c|c|c|c|}
\hline & Group Non-obese $(n=59)$ & Group Obese $(n=35)$ & Total $(\mathrm{n}=94)$ & P value \\
\hline VAS score $>3$ patients, $\mathrm{n}(\%)$ & & & & $0.014^{\mathrm{a}}$ \\
\hline No & $42(71.2)$ & $16(45.7)$ & $58(61.7)$ & \\
\hline Yes & $17(28.8)$ & $19(54.3)$ & $36(38.3)$ & \\
\hline Pain VAS score-T0 & $3[2$ to 4$]$ & $4[3$ to 5$]$ & $3[3$ to 5$]$ & $0.026^{c}$ \\
\hline Pain VAS score-T1 & $2[2$ to 3$]$ & $2[2$ to 3$]$ & $2[2$ to 3$]$ & $0.009^{c}$ \\
\hline Pain VAS score-T2 & $2[1$ to 2$]$ & $2[2$ to 2$]$ & $2[1$ to 2$]$ & $0.009^{c}$ \\
\hline Pain VAS score-T3 & $2[1$ to 2$]$ & $2[2$ to 2$]$ & $2[1$ to 2$]$ & $0.139^{c}$ \\
\hline Pain VAS score-T4 & $2[1$ to 2$]$ & $2[2$ to 3$]$ & $2[1$ to 2$]$ & $0.068^{c}$ \\
\hline $\begin{array}{l}\text { Patients requiring fentanyl } \\
\text { bolus intraoperatively, }(\%)\end{array}$ & $33(55.9)$ & $27(77.1)$ & $60(63.8)$ & $0.039^{\mathrm{a}}$ \\
\hline Total tramadol consumption & $16.10(34.0)$ & $40.0(46.6)$ & $25(40.6)$ & $0.003^{b}$ \\
\hline Nausea & & & & $0.047^{a}$ \\
\hline No & $53(89.8)$ & $26(74.3)$ & $79(84.0)$ & \\
\hline Yes & $6(10.2)$ & $9(25.7)$ & $15(16.0)$ & \\
\hline Vomiting & & & & $0.062^{\mathrm{a}}$ \\
\hline No & $58(98.3)$ & $31(88.6)$ & $89(94.7)$ & \\
\hline Yes & $1(1.7)$ & $4(11.4)$ & $5(5.3)$ & \\
\hline
\end{tabular}




\begin{tabular}{|c|c|c|c|c|c|c|}
\hline & BMI & 1 & 2 & 3 & 4 & 5 \\
\hline 1. VAS-T0 & $.207^{\star}$ & 1 & & & & \\
\hline 2. VAS-T1 & $.287^{\star *}$ & $.749^{\star *}$ & 1 & & & \\
\hline 3. VAS-T2 & $.206^{\star}$ & $.538^{* *}$ & $.660^{\star *}$ & 1 & & \\
\hline 4. VAS-T3 & .171 & $.379^{* *}$ & $.480^{\star *}$ & $.740^{\star *}$ & 1 & \\
\hline 5. VAS-T4 & .134 & $.381^{\star *}$ & $.492^{\star *}$ & $.595^{\star *}$ & $.735^{\star *}$ & 1 \\
\hline $\begin{array}{l}\text { 6. Total } \\
\text { tramadol } \\
\text { consumption }\end{array}$ & $.307^{\star *}$ & $.487^{\star *}$ & $.476^{\star *}$ & $.448^{\star *}$ & $.257^{\star}$ & $.370^{\star *}$ \\
\hline
\end{tabular}

\section{DISCUSSION}

The aim of this retrospective study was to investigate the relationship between BMI and postoperative pain scores and analgesic consumption in patients who underwent LC. Several important findings were identified in the present study. First; postoperative pain scores were higher in obese patients. Second; intraoperative opioid requirement and total opioid consumption were higher in obese patients. Finally; a significant correlation was found between BMI scores and postoperative pain scores and opioid consumption.

Acute postoperative pain is defined as pain of rapid onset and short duration resulting from nociceptive stimulation of tissues induced by operative stress. Pain sensitivity is highest during the first 24 hours postoperatively and gradually decreases over this period (8). Despite increasing knowledge and experience in the treatment of acute postoperative pain, acute pain after laparoscopic surgery is still a major problem (5). It is known that the incidence of postoperative pain after laparoscopic surgery can be up to $70 \%$. In our study, in accordance with the literature, approximately $38.3 \%$ of the patients in the postoperative recovery unit had VAS pain scores $>3$.

Many previous studies have examined the relationship between BMI and various pain syndromes $(6,9)$. These studies have shown that high BMI is a risk factor for perceived pain (6). In a meta-analysis in which 53,362 patients were examined and 33 studies were included, it was revealed that higher BMI may be a predictor of poorly controlled acute postoperative pain (6). In the same study, young age, female gender, smoking, history of depressive symptoms, history of anxiety symptoms, sleep difficulties, and use of preoperative analgesia were also reported as other risk factors (6). In our study, the number of patients with VAS $>3$ at the T- 0 time point was found to be higher in the obese group. However, pain scores were higher in the obese group during the first 12 hours. In addition, in the study of Elgendy et al. (10), the analgesic requirements of morbidly obese and supermorbid patients who underwent laparoscopic sleeve gastrectomy were compared. In this study, recovery unit tramadol consumption and total paracetamol consumption showed significant differences between the groups. Moreover, postoperative pain scores and hemodynamic stability values were similar between the groups. In another past study, in ambulatory surgery patients; BMI, duration of anesthesia, and certain types of surgery were found to be important determinants of pain in the post-anesthesia care unit (11). Based on these results, high BMI may also be a risk factor for the presence of postoperative pain in LC patients. These evidences in the literature strengthen our hypothesis that there is a relationship between postoperative pain and BMI levels.

When the literature is examined in detail, there are also studies reporting that BMI is not associated with postoperative pain and opioid consumption $(7,12)$. Cristina et al. analyzed the relationship of BMI and some serum tissue damage markers with postoperative pain in a study they conducted in patients undergoing inguinal hernia surgery. In this study, contrary to what is known, the authors reported that higher BMI is not associated with higher postoperative pain and that high serum LDH levels can provide useful information to predict moderate to severe postoperative pain (12). Similarly, Grodofsky et al. (7) in a study conducted in patients undergoing ankle fracture surgery, aimed to determine the relationship between gender and BMI and acute postoperative pain scores. In this study, patients received only morphine as preemptive analgesia, and no association was found between obesity or gender and postoperative pain (7).

Although the physiological mechanism of the relationship between obesity and pain has not yet been clearly explained, various hypotheses have been put forward. When the studies are examined in detail, there are limited studies in this field and the evidence is insufficient. In the literature, obesity-related pain has been studied from three aspects: mechanical (mechanical pressure on tissue and joint), behavioral (sleep and physical activity), and physiological (inflammatory theory) $(13,14)$. It has been reported that obesity is basically a low-grade inflammatory disease and obesity-related pain is associated with a systemic inflammatory state. It has been reported that modulators such as proinflammatory cytokines (TNF- $\alpha$, IL-6, IL$1 \beta$, and leptin), ghrelin, and galanin cause an increase in pain sensitivity in patients with obesity (13-16). All these mechanisms in the literature show that BMI is associated with various types of pain.

Another result of this study was that patients who needed intraoperative fentanyl were more common in the obese group. Our results are consistent with studies in the literature revealing the relationship between BMI and pain (6,9,13-16). Unlike our study, Elgendy et al. found that intraoperative fentanyl and morphine consumption was lower in super morbidly obese patients. As the reason 
for this result; reported that since obese patients are more sensitive to opioids, the application of effective multimodal analgesia in their institute may contribute to reducing the intraoperative or postoperative opioid requirements (10). Pain regulation has been associated with endogenous opioids in the literature. However, in an experimental study, it was reported that basal endogenous opioid levels were higher in obese mice (17). On the other hand, Elderly et al. also claimed in their study that less need for analgesia in super-obese patients may be due to higher endogenous endorphin levels in obese patients (10).

Management of postoperative pain in obese patients is specific. Postoperative pain management should be multimodal to provide effective postoperative analgesia, facilitate physiotherapy and mobilization, and avoid opioid-related side effects. Paracetamol can be used safely for this purpose, but its effectiveness is limited (18). In our study, it was determined that the patients routinely received IV paracetamol twice a day according to the clinical protocol. Despite this treatment protocol, it was observed that especially obese patients consumed more opioids. NSAIDs are recommended for multimodal analgesia because of better analgesia, reduced opioid consumption, and fewer side effects (18).

One of the results of our study; obese patients have a higher incidence of nausea. These results are consistent with previous published studies. Nausea-vomiting is an important problem frequently encountered in the acute postoperative period. The causes of this problem may be due primarily to obesity or the high doses of opioids used more in obese patients. It is known that side effects of opioids such as nausea and vomiting are common $(19,20)$. It has been reported in the literature that increased BMI is an important risk factor for postoperative nausea and vomiting (19). Conversely, there is also a study reporting that increased BMI is not a risk factor for postoperative nausea and vomiting (20).

This study has some limitations. Retrospective design, single-center design, small study population, short postoperative follow-up, and reliance on subjective pain scores are some of the limitations of this study.

\section{CONCLUSION}

According to our conclusion from this study, BMI is associated with acute postoperative pain and obese patients need more postoperative opioid analgesia. For this reason, it is necessary to determine the need for analgesia in patients who will undergo laparoscopic cholecystectomy in the acute postoperative period, after taking into account the obesity status. Our results should be supported by multicenter, randomized, and large patient series.

\section{ETHICAL DECLARATIONS}

Ethics Committee Approval: The study was approved by the Yozgat Bozok University Clinical Researchs Ethics Committee (Date: 27.01.2021, Decision No: KAEK189_2021.01.27_05).

Informed Consent: Because the study was designed retrospectively, no written informed consent form was obtained from patients.

Referee Evaluation Process: Externally peer-reviewed.

Conflict of Interest Statement: The authors have no conflicts of interest to declare.

Financial Disclosure: The authors declared that this study has received no financial support.

Author Contributions: All of the authors declare that they have all participated in the design, execution, and analysis of the paper, and that they have approved the final version.

Acknowledgement: The authors would also like to thank all of the staff in the Department of Anesthesiology and General Surgery for their fundamental support for this study (Yozgat Bozok University, Medical Faculty Hospital, Yozgat, Turkey).

\section{REFERENCES}

1. Wills VL, Hunt DR. Pain after laparoscopic cholecystectomy. Br J Surg 2000; 87: 273-84.

2. Bisgaard T, Klarskov B, Rosenberg J, Kehlet H. Characteristics and prediction of early pain after laparoscopic cholecystectomy. Pain 2001; 90: 261-9.

3. Karanikolas M, Swarm RA. Current trends in perioperative pain management. Anesthesiol Clin North Am 2000; 18: 575-99.

4. Liu Q, Ji MH, Dai YC, et al. Predictors of acute postsurgical pain following gastrointestinal aurgery: a prospective cohort study. Pain Res Manag 2021; 2021:6668152

5. Wright LJ, Schur E, Noonan C, Ahumada S, Buchwald D, Afari N. Chronic pain, overweight, and obesity: findings from a community-based twin registry. J Pain 2010; 11: 628-35.

6. Yang MMH, Hartley RL, Leung AA, et al. Preoperative predictors of poor acute postoperative pain control: a systematic review and meta-analysis. BMJ Open 2019; 9: e025091.

7. Grodofsky SR, Sinha AC. The association of gender and body mass index with postoperative pain scores when undergoing ankle fracture surgery. J Anaesthesiol Clin Pharmacol 2014; 30: 248-52.

8. Vergés MTS, Puigbò EC, Cortada MG, Rizo MR, Garcia MVM. New trends in the treatment of post-operative pain in general and gastrointestinal surgery. Cir Esp 2009; 86: 63-71.

9. Ip HYV, Abrishami A, Peng PWH, Wong J, Chung F. Predictors of postoperative pain and analgesic consumption: a qualitative systematic review. Anesthesiology 2009; 111: 657-77.

10. Elgendy H, Youssef T, Banjar A, Elmorsy S. Decreased analgesic requirements in super morbidly versus morbidly obese patients undergoing laparoscopic sleeve gastrectomy. Obes Surg 2020; 30: 2715-22.

11. Chung F, Ritchie E, Su J. Postoperative pain in ambulatory surgery. Anesth Analg 1997; 85: 808-16. 
12.González-Callejas C, Aparicio VA, De Teresa C, Nestares T. Association of body mass index and serum markers of tissue damage with postoperative pain. the role of lactate dehydrogenase for postoperative pain prediction. Pain Med 2020; 21: 1636-43.

13. Chin SH, Huang WL, Akter S, Binks M. Obesity and pain: a systematic review. Int J Obes 2020; 44: 969-79.

14.Zahorska-Markiewicz B, Kucio C, Pyszkowska J. Obesity and pain. Hum Nutr Clin Nutr 1983; 37: 307-10.

15. Janke EA, Collins A, Kozak AT. Overview of the relationship between pain and obesity: what do we know? where do we go next? J Rehabil Res Develop 2007; 44: 245-62.

16. Hitt HC, McMillen RC, Thornton-Neaves T, Koch K, Cosby AG. Comorbidity of obesity and pain in a general population: results from the southern pain prevalence study. J Pain 2007; 8: 430-6.

17. Khawaja XZ, Chattopadhyay AK, Green IC. Increased $\beta$-endorphin and dynorphin concentrations in discrete hypothalamic regions of genetically obese mice. Brain Res 1991; 555: 164-8.

18. Schug SA, Raymann A. Postoperative pain management of the obese patient. Best Pract Res Clin Anaesthesiol 2011; 25: 73-81.

19. Watcha MF, White FP. Postoperative nause and womiting. Anesthesiology 1992; 77: 162-84.

20. Kranke P, Apfel CC, Papenfuss T, et al. An increased body mass index is no risk factor for postoperative nausea and vomiting. a systematic review and results of original data. Acta Anaesthesiol Scand 2001; 45: 160-6. 